\title{
Zinc causes the death of hypoxic astrocytes by inducing ROS production through mitochondria dysfunction
}

\author{
Rong Pan ${ }^{1 凶}$, Ke Jian Liu' ${ }^{1}$ Zhifeng $Q i^{2 \bowtie}$ \\ 1 Department of Pharmaceutical Sciences, University of New Mexico, Albuquerque, NM 87131-0001, USA \\ 2 Cerebrovascular Diseases Research Institute, Xuanwu hospital of Capital Medical University, Beijing 100053, China
}

Received: 16 January 2019 / Accepted: 20 June 2019 / Published online: 25 September 2019

\begin{abstract}
Cerebral ischemia triggers a cascade of events that contribute to ischemic brain damages. Zinc release and accumulation has been shown to lead to brain cell death following cerebral ischemia. However, the mechanism underlying remains to be elucidated. Our recently published work showed that suppression of mitochondrial-derived reactive oxygen species (ROS) production significantly reduced ischemic stroke related brain damage within $6 \mathrm{~h}$. Herein, we investigated the relationship between zinc accumulation and mitochondrial-derived ROS production in astrocytes after 3-h hypoxia. We found that inhibition of mitochondrial-derived ROS significantly decreased total amount of ROS generation and cell death in primary astrocytes during hypoxia when zinc was overload. In contrast, the inhibition of NADPH oxidase-derived ROS had less of an effect. Our results also showed that zinc and mitochondria were colocalized in hypoxic astrocytes. Moreover, extracellular zinc addition caused zinc accumulation in the mitochondria and decreased mitochondrial membrane potential, leading to mitochondria dysfunction. These findings provide a novel mechanism that zinc accumulation contributes to hypoxiainduced astrocytes death by disrupting mitochondria function, following cerebral ischemia.
\end{abstract}

Keywords Hypoxia, Zinc, Mitochondria, Reactive oxygen species

\section{INTRODUCTION}

Zinc is essential for cerebral development, which plays a signaling role in the central nerve system (Bitanihirwe and Cunningham 2009). However, the pathological release of zinc can be highly toxic as it causes brain cell death through protein aggregation and direct neurotoxicity (Weiss et al. 2000). Our previous study has shown that ischemic stroke induces an abnormally high concentration of zinc in neurons, leading to brain cell death (Zhao et al. 2014). However, the underlying mechanism remains to be fully elucidated.

Besides excessive intracellular accumulation of zinc, reactive oxygen species (ROS) generation has long been

$\square$ Correspondence: rpan@salud.unm.edu (R. Pan), email_qzf@163.com (Z. Qi) recognized as another important factor that causes brain damages after cerebral ischemia (Zhao et al. 2018a). ROS are generated in the normal condition (Poli et al. 2004). However, at high concentrations, ROS are cytotoxic, causing protein oxidation and DNA damage (Olmez and Ozyurt 2012). ROS are generated through multiple diverse sources, including NADPH oxidase, mitochondria, cyclooxygenase, and monoamine oxygenase (Abramov et al. 2007; Adibhatla and Hatcher 2010; Yang et al. 2018). Among these sources, NADPH oxidase has been considered as a major pathway to generate neuronal ROS, which causes cell death in hypoglycemia as well as in anoxia and reoxygenation (Abramov et al. 2007). Notably, we recently reported that suppressing the production of mitochondrial-derived ROS by mitochondria-targeted ROS inhibitor $\mathrm{R}(+)$ pramipexole $[R(+)$ PPX] significantly reduced brain damage at $6 \mathrm{~h}$ after the cerebral ischemia onset (Zhao et al. 2018b). 
Our finding indicates that mitochondrial-derived ROS is involved in brain cell death at the early stage of ischemic stroke. Mitochondria generate a huge amount of ATP to maintain the homeostasis of neuron. However, mitochondria also are a major producer of ROS, as well as the key activators of programmed cell death (Nicholls and Budd 2000). Overloading of zinc triggers the loss of mitochondrial membrane potential, and zinc can cause mitochondrial dysfunction in all physiologically relevant substrate conditions (Dineley et al. 2005). The dysfunctional mitochondria enhances ROS generation, which leads to apoptosis (Federico et al. 2012). Moreover, the good co-localization of zinc and ROS further indicates a close relationship between zinc and ROS (Zhao et al. 2018b). Thus, we speculate that zinc may cause brain cell death through mitochondrial-derived ROS generation at early phase of ischemic stroke.

In the present study, we tested the hypothesis that high concentrations of zinc cause mitochondrial dysfunction, leading to excess mitochondria-derived ROS production, which promotes brain cell death during ischemia. To investigate the interaction between zinc and mitochondria-derived ROS at early phase of ischemic stroke, ROS production was measured following $3 \mathrm{~h}$ of hypoxia with or without zinc addition. We also investigated whether decreasing ROS level by ROS scavenger, mitochondria-targeted ROS inhibitor, or NADPH oxidase inhibitor would reduce the total ROS level and astrocytic death after hypoxic treatment. Moreover, we investigated whether zinc addition causes mitochondrial dysfunction and ROS generation.

\section{RESULTS}

\section{Astrocytes protect neurons against the toxicity of zinc under hypoxic condition}

Studies show that following ischemic stroke, astrocytic death occurs prior to neuronal death (Liu et al. 1999; Ouyang et al. 2007). Hypoxia is the hallmark of ischemic stroke. Astrocytes and neurons were cocultured to investigate the effects of hypoxia on them. As shown in Fig. 1, the neuronal death rate was much higher than astrocytic death rate under normoxia condition. However, astrocytic death rate was markedly higher than neuronal death rate under hypoxia condition. It suggests that astrocytes are more vulnerable by hypoxia and neurons have been protected by astrocytes under hypoxia.

\section{Zinc augments hypoxia-induced ROS generation}

Our results suggest that the death of astrocytes protects neurons. Thus, investigating the mechanism of zincinduced astrocytes death is important to understand the mechanism of ischemic stroke-induced injury. ROS is a major effector in ischemic cell death. Moreover, we recently reported a significant increase in level of ROS, which colocalized with accumulated zinc in ischemic rats' brain. Furthermore, suppressing the production of mitochondrial-derived ROS significantly reduced brain damage at $6 \mathrm{~h}$ following the onset of ischemic stroke (Zhao et al. 2018b). Thus, we wanted to know whether ROS is involved in zinc-induced astrocytic death in hypoxia. ROS level was first measured under different conditions using immuno-spin trapping. Immuno-spin trapping is a sensitive and specific method to detect macromolecule derived radicals (Ramirez and Mason 2005; Towner et al. 2012). Here we used DMPO as the spin trap for ROS. The ROS level slightly increased after $3 \mathrm{~h}$ of treatment with hypoxia or with $100 \mu \mathrm{mol} / \mathrm{L}$ zinc alone, while combination of these two factors dramatically increased ROS generation (Fig. 2). It indicates that zinc promotes hypoxia-induced ROS generation. Notably, chelation of zinc by its specific chelator, $N, N, N^{\prime}, N^{\prime}$ Tetrakis(2-pyridylmethyl)ethylenediamine (TPEN, $1 \mu \mathrm{mol} / \mathrm{L}$ ), significantly blocked ROS generation. These data suggest that zinc promotes ROS generation in hypoxic astrocytes.

\section{Mitochondria are a major source of ROS generation in zinc-treated hypoxic astrocytes}

To investigate the sources of ROS generation in zinctreated hypoxic cells, cell permeable SOD mimetic MnTMPyP $(100 \mu \mathrm{mol} / \mathrm{L})$, NADPH oxidase inhibitor DPI $(10 \mu \mathrm{mol} / \mathrm{L})$ and mitochondrial-target antioxidant PPD (1 mmol/L) were used to inhibit different sources of ROS generation. We found that as expected, MnTMPyP reduced the production of ROS most significantly, since MnTMPyP removes ROS that are generated from all sources. Importantly, PPD decreased ROS generation more than DPI (Fig. 3), indicating that mitochondria served as a main source of zinc-augmented ROS generation in astrocytes during hypoxia.

\section{ROS is involved in zinc-induced astrocytic death}

Next, we used ROS scavengers to further investigate the role of ROS in zinc-mediated hypoxic astrocytes death. MnTMPyP decreased cellular death in hypoxic 
Fig. 1 Astrocyte protected neuronal against zinc under hypoxia. Primary astrocytes were seeded on the inserts and neurons were plated on wells. After the neurons had adhered to the wells, the inserts with astrocytes were placed into the wells and exposed to 3-h hypoxia or normoxia with indicated concentration of zinc.

A Astrocytes on the cell culture inserts were stained by GFAP antibody while neurons on the wells were stained by NeuN antibody. Scale bar $200 \mu \mathrm{m}$. B Cell death rates $(n=3)$. Data were presented as mean \pm SEM. ${ }^{*} p<0.05$ versus the normoxia group with same zinc concentration; ${ }^{\#} p<0.05$ versus the same group (normoxia or hypoxia) without zinc treatment
A

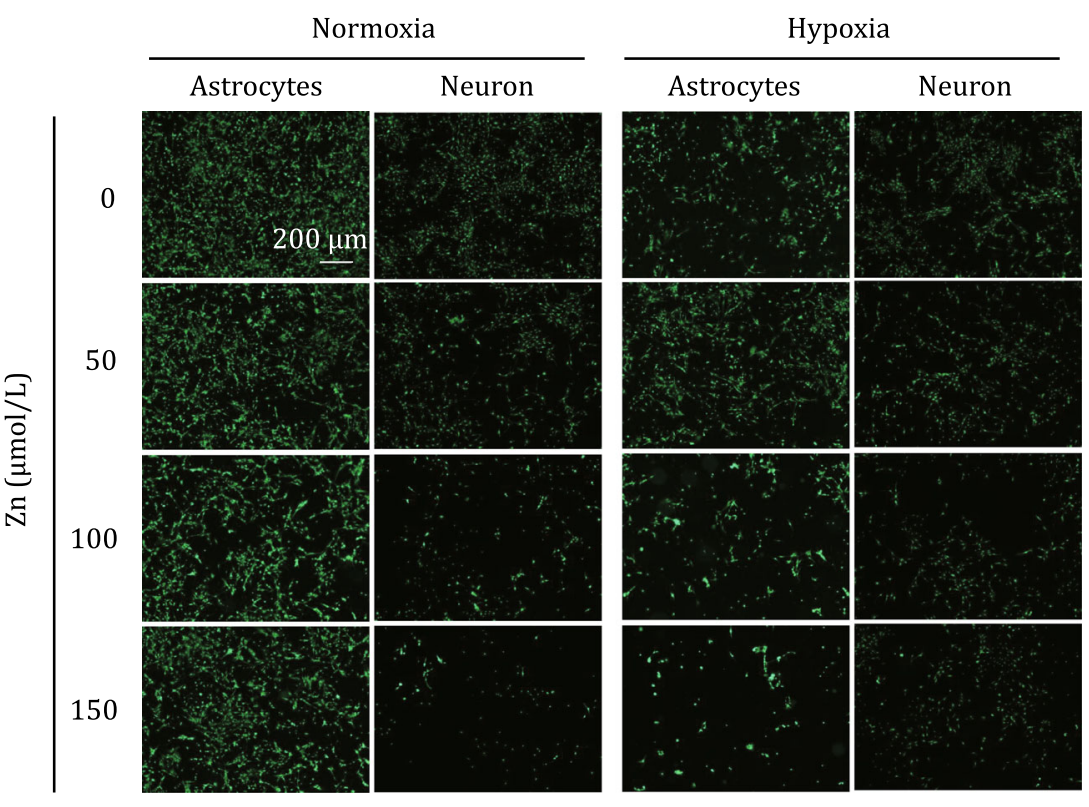

B

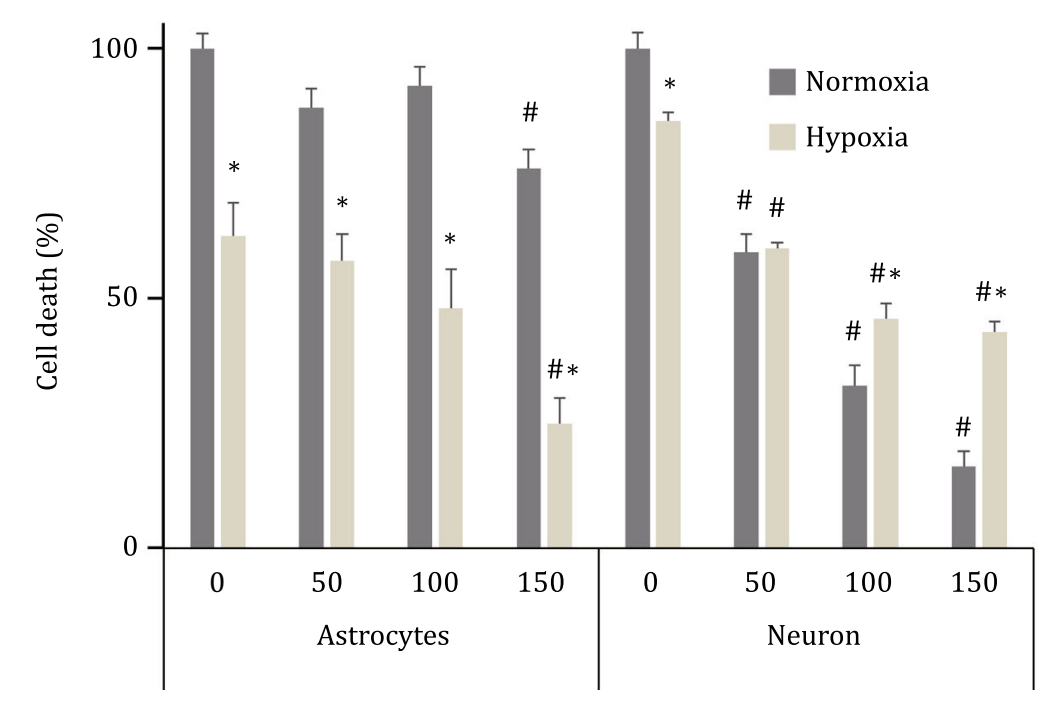

astrocytes with zinc treatment (Fig. 4), confirming that ROS participates in the zinc-induced astrocytic death in hypoxia. Moreover, DPI and PPD were used to block different sources of ROS. As shown in Fig. 4, PPD was more effective in presenting zinc-induced hypoxic astrocytic death than DPI. These findings indicate that mitochondria-initialed ROS, a major source of ROS generation, contributes more than NADPH oxidase generated ROS to zinc-induced hypoxic astrocytic death.

\section{Zinc accumulation at mitochondria in hypoxic astrocytes}

The above results demonstrate that zinc promotes ROS generation mainly through mitochondria. Therefore, we next wanted to determine how zinc increases mitochondria-derived ROS generation. The specific probes for zinc (Fluorzin-3) and mitochondria (Mito-tracker) were utilized to localize zinc and mitochondria in the cells. Our results show that mitochondria concentrated around the nucleus, colocalizing with high concentration of zinc in the zinc-treated hypoxic astrocytes (Fig. 5). The colocalization of zinc and mitochondria suggests that zinc and mitochondria may interact with each other. To further confirm that zinc interacts with mitochondria, mitochondria were isolated from normoxic and hypoxic astrocytes. Then, zinc was stained with FluoZin-3 zinc probe. Zinc levels in mitochondria were assessed by calculating the density of FluoZin-3 signal through fluorescence spectrometer. As shown in Fig. 6, zinc level significantly increased after hypoxia plus zinc treatment, while there was hardly any 
Fig. 2 Zinc-augmented ROS generation in hypoxic astrocytes. Primary astrocytes were exposed to 3-h hypoxia or normoxia with or without zinc treatment $(100 \mu \mathrm{mol} / \mathrm{L})$. Zinc chelate TPEN $(1 \mu \mathrm{mol} / \mathrm{L})$ was used to investigate the role of zinc. A DMPO bound macromolecular radical levels in astrocytes were measured by immuno-spin trapping (green), while nucleus was stained by DAPI (blue). Scale bar $30 \mu \mathrm{m}$. B Immune-spin trapping fluorescence $(n=3)$. Data were presented as mean \pm SEM. ${ }^{*} p<0.05$, ${ }^{* *} p<0.01$ versus the normoxia group; ${ }^{\# \#} p<0.01$ versus the hypoxia and zinc treatment group
A

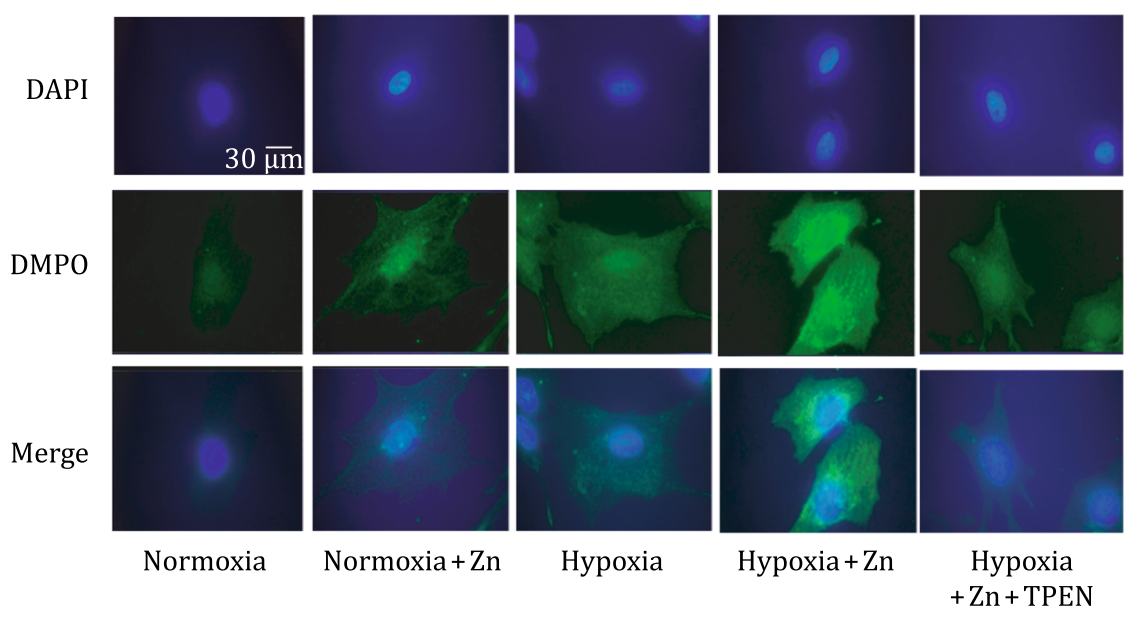

B

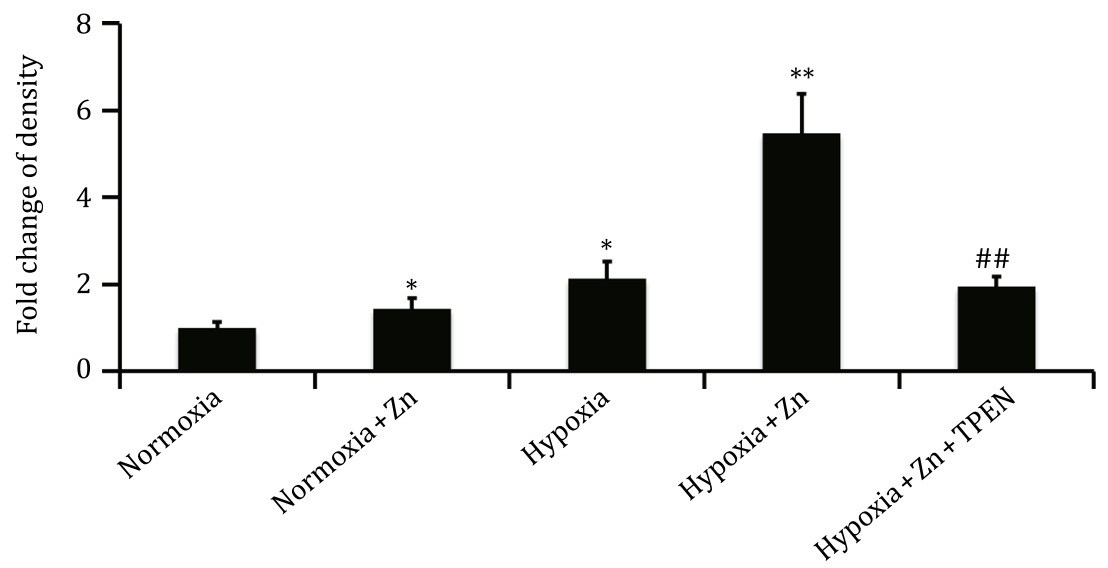

uptake of zinc by mitochondria under normoxia. These results demonstrate that hypoxia promotes mitochondria to uptake zinc and that zinc only accumulates in mitochondria of hypoxic but not normoxic astrocytes.

\section{Zinc accumulation causes mitochondria dysfunction}

The loss of mitochondrial membrane potential is the reason for ROS release from mitochondria (MartinezReyes et al. 2016). The vital mitochondrial dye JC-1, undergoing a reversible change in fluorescence emission from red to green as mitochondrial membrane potential decreases, has been used to measure mitochondrial function. As shown in Fig. 7, under the condition of hypoxia with zinc overload, mitochondrial membrane potential decreased markedly. Mitochondria morphology was changed from thread to round. These results suggest that mitochondrial membrane potential has been altered by zinc under hypoxic condition, leading to excess ROS release and further causes hypoxic astrocytic death.

\section{DISCUSSION}

The present study investigated the interaction between zinc and ROS in acute hypoxic astrocytes, and explored the relationship of zinc accumulation and mitochondria dysfunction. We demonstrated that mitochondrialderived ROS is a major source of ROS generation in acute ( $3 \mathrm{~h}$ ) hypoxic astrocytes. Notably, zinc accumulation in mitochondria caused mitochondrial dysfunction, which is a major reason for ROS over-generation in hypoxic astrocytes.

Following ischemic stroke, zinc releases from glutamatergic terminal into surrounding milieu and is then taken up by neighboring cells (Galasso and Dyck 2007). Other researchers and our group clearly demonstrate that intracellular zinc accumulation contributes significantly to brain injury after ischemia (Filipiak et al. 2010; Hamatake et al. 2000; Pan et al. 2013, 2015; Zhao et al. 2014). Thus, understanding the mechanism of zincinduced ischemic cell death is critical for ischemic stroke research. Besides zinc, ROS generation has been 
Fig. 3 The sources of ROS generation in hypoxic astrocyte after zinc treatment. Astrocytes were exposed to 3-h hypoxia following $100 \mu \mathrm{mol} / \mathrm{L}$ zinc and MnTMPyP (100 $\mu \mathrm{mol} / \mathrm{L})$, DPI $(10 \mu \mathrm{mol} / \mathrm{L})$ or PPD $(1 \mathrm{mmol} / \mathrm{L})$ treatment. A DMPO bound macromolecular radical levels in astrocytes were measured by immuno-spin trapping (green). Nucleus was stained by DAPI (blue). Scale bar $30 \mu \mathrm{m}$. B Immune-spin trapping fluorescence $(n=3)$. Data were presented as mean \pm SEM. ${ }^{* *} p<0.01$ versus the hypoxia and zinc treatment group
A



Hypoxia + Zn


Hypoxia $+\mathrm{Zn}$ + MnTMPyP

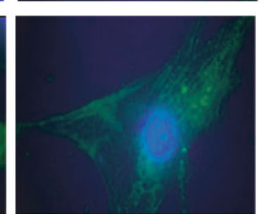

Hypoxia $+\mathrm{Zn}+\mathrm{PPD}$

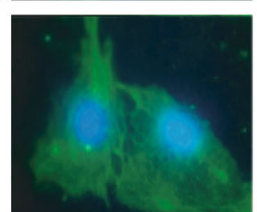

Hypoxia $+\mathrm{Zn}+\mathrm{DPI}$

B

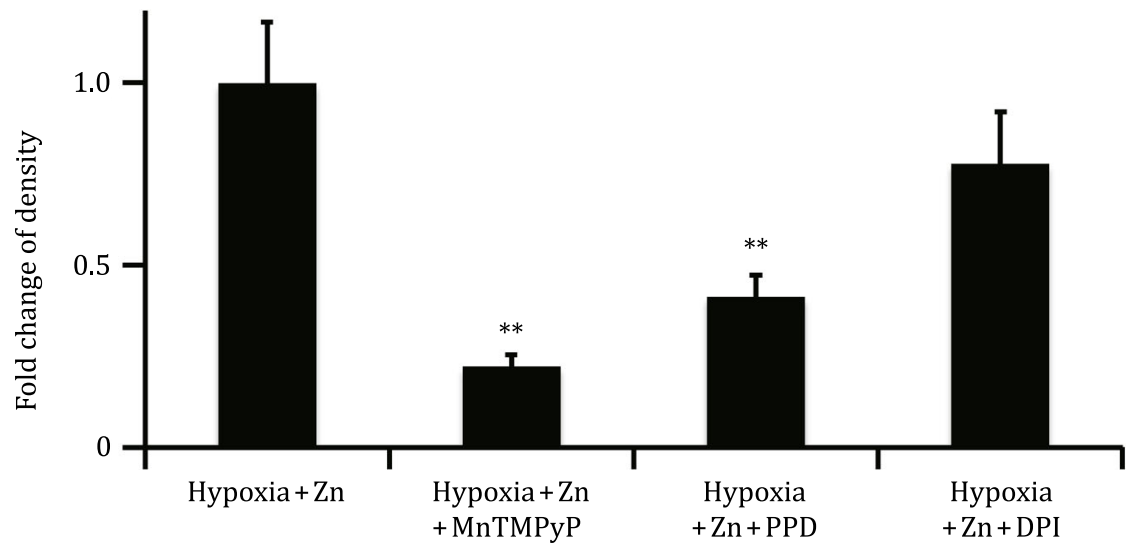

recognized as a key factor, leading to brain cell death in ischemic stroke (Piantadosi and Zhang 1996). Our data showed that zinc promotes ROS production in hypoxic astrocytes. Notably, although $100 \mu \mathrm{mol} / \mathrm{L}$ zinc or $3 \mathrm{~h}$ hypoxia alone did not induce significant ROS generation, the synergism of these two treatments dramatically increased ROS generation (Fig. 2), which contributes to increased astrocytic death under hypoxic conditions (Fig. 4). Then, the chelation of zinc by its specific chelator TPEN significantly decreased ROS generation in astrocytes after $3 \mathrm{~h}$ hypoxia exposure (Fig. 2), which confirms that zinc accumulation is the cause of ROS generation in hypoxic astrocytes. Moreover, we found that the removal of ROS remarkably decreased zincinduced hypoxic astrocytic death (Fig. 4). Ischemic stroke causes decreased blood flow, resulting in reduction of tissue oxygenation. Our previous in vivo electron paramagnetic resonance (EPR) study showed that cerebral tissue oxygenation decreased from $33.4 \pm 6.0$ to $1.2 \pm 0.7 \mathrm{mmHg}$ in the ischemic core region, which clearly indicates that ischemic tissue is a hypoxic environment (Liu et al. 2004). As a main feature of ischemic stroke, hypoxia plays a fundamental role in ischemic stroke-caused mortality (Semenza et al. 2000). Our data demonstrate that zinc-induced ROS production is involved in the hypoxic brain cell death in the early ischemic stroke.

In the traditional view, NADPH oxidase is the major source of neuronal ROS generation, causing cell death in hypoglycemia, as well as in anoxia and reoxygenation (Abramov et al. 2007; Suh et al. 2007). However, our results show that mitochondria-derived ROS production is a major source of ROS at the first 3-h hypoxic in the present of zinc (Fig. 3). Furthermore, our results show that mitochondrial-target antioxidant decreased zincinduced hypoxic primary astrocytic death while NADPHoxidase inhibitor had much lesser effect in preventing zinc-induced cell death (Fig. 4). These findings imply that mitochondria-derived, but not NADPH-initiated ROS production is a major source of ROS generation in 


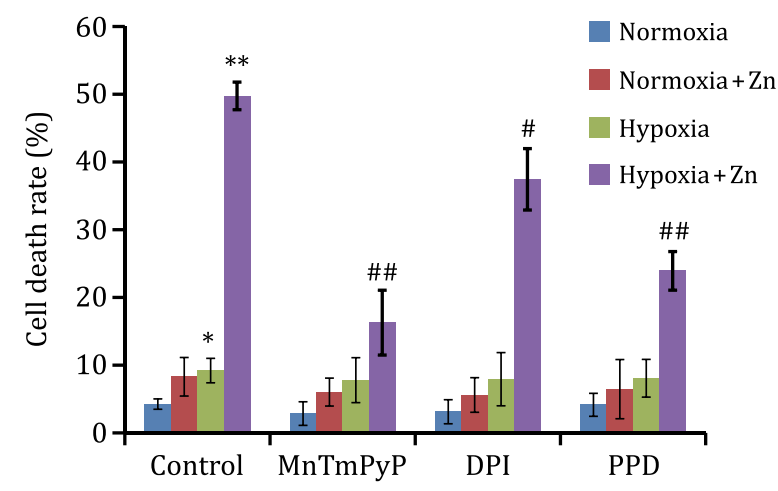

Fig. 4 Effects of ROS on zinc-induced cell death. Before exposure to hypoxia/normoxia, primary astrocytes were pretreated with MnTMPyP $(100 \mu \mathrm{mol} / \mathrm{L})$, DPI $(10 \mu \mathrm{mol} / \mathrm{L})$ or PPD $(1 \mathrm{mmol} / \mathrm{L})$ with or without $100 \mu \mathrm{mol} / \mathrm{L}$ zinc for $1 \mathrm{~h}$. Cell viability was assayed after 3 -h exposure. ${ }^{*} p<0.05,{ }^{* *} p<0.01$ compared with the normoxia group; ${ }^{\#} p<0.05,{ }^{\# \#} p<0.01$ compared with the hypoxia and zinc treatment group

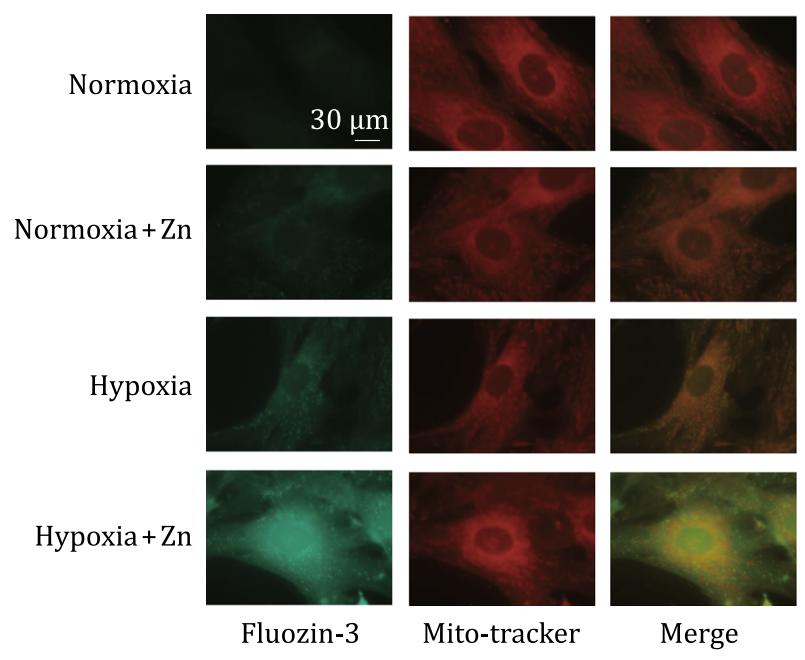

Fig. 5 Co-localization of zinc and mitochondria. Primary astrocytes were exposed to hypoxia or normoxia for $3 \mathrm{~h}$ with or without zinc treatment $(100 \mu \mathrm{mol} / \mathrm{L})$. The intracellular-free zinc was visualized using FluoZin-3 (green). Mitochondria were stained by Mito-tracker (red). Scale bar $30 \mu \mathrm{m}$

early ischemia, and thus is a major contributing factor to the brain damage during early ischemic stroke. This is consistent with our recent report that mitochondrial ROS production is dominant at $6 \mathrm{~h}$ after reperfusion in stroke rats (Zhao et al. 2018b). Interestingly, our published study has also shown that NADPH-initiated ROS is the major source of ischemia/reperfusion-induced ROS, especially after $24 \mathrm{~h}$ reperfusion, which corroborates the traditional view (Zhao et al. 2018b). Together, these diverse findings reveal that the major sources of ROS production, whether NADPH, mitochondrial or others, are dependent on the ischemic and/or reperfusion time.



Fig. 6 Zinc concentration detection in mitochondria. Primary astrocytes were exposed to 3-h hypoxia or normoxia in the presence or absent of $100 \mu \mathrm{mol} / \mathrm{L}$ zinc. Then mitochondria were isolated by Mitochondria Isolation Kit for Cultured Cells. The level of zinc in mitochondria was measured by mixing with FluoZin-3 fluorescence probe and reading the fluorescence intensity. ${ }^{*} p<0.05$ versus the normoxia group

Mitochondria generating massive of ATP and limiting the production of ROS are necessary for maintaining cellular homeostasis and function. However, mitochondria can promote cellular death by releasing proapoptotic factors such as cytochrome $\mathrm{C}$ and apoptosis inducing factor (AIF) under stress. Mitochondrial disorder is thought as a key incident in numerous neurodegenerative diseases, including stroke (Nicholls and Budd 2000; Zamzami and Kroemer 2001). It was proved that zinc blocks the cellular energy generation through disruption of mitochondria function (Dineley et al. 2003). One report shows that zinc may concentrate at the bc1 complex and inhibit electron transport there (Berry et al. 2000). However, the relationship between zinc and mitochondria after ischemia is unclear. We first noticed, in this study, that zinc and mitochondria are colocalized in hypoxic astrocytes (Fig. 5). By measuring zinc level in the isolated mitochondria, we demonstrated that zinc is accumulated in mitochondria (Fig. 6), which provides direct evidence on the interaction of zinc and mitochondria in acute hypoxic astrocytes. Although our earlier reports have shown that zinc accumulates intracellularly in hypoxic astrocytes (Pan et al. 2013; Pan and Liu 2016), the present study zoomed in on mitochondria, and found that the zinc accumulation is intra-mitochondrial. This is the first evidence for locating the accumulated intracellular zinc at an organelle in the hypoxic astrocytes, which helps us to better understand how zinc causes hypoxic cellular damage. Furthermore, by assessing the membrane potential of mitochondria, we found zinc significantly decreased membrane potential and altered the 
A
Normoxia

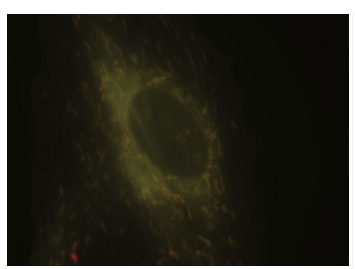

Hypoxia

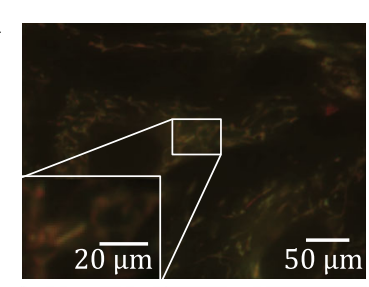



Normoxia $+\mathrm{Zn}$



Hypoxia $+\mathrm{Zn}$
B



Fig. 7 Zinc caused mitochondria dysfunction. Mitochondrial function was assessed by JC- 1 staining, measuring the membrane potential. A Red to green means mitochondrial membrane potential decrease. B The rate of green and red signal intensity $(n=3)$. Data were presented as mean \pm SEM. ${ }^{*} p<0.05$, ${ }^{* *} p<0.01$ versus the normoxia group. Scale bar for the whole cells is $50 \mu \mathrm{m}$. Scale bar for the enlarged region is $20 \mu \mathrm{m}$

morphology (Fig. 7) of mitochondria in hypoxic astrocytes. Since mitochondrial membrane potential depression is the reason of ROS release from mitochondria, our results suggest that ROS in acute hypoxic astrocytes is caused by zinc-induced mitochondria dysfunction. Findings from the present study and our reports reveal a mechanism of ischemic stroke-induced brain damage that ischemia induces an increase of intracellular zinc, which causes mitochondrial dysfunction, leading to ROS generation and brain damage. This zinc-mitochondriaROS pathway is likely a key mechanism of zinc-induced astrocytic death following ischemia.

\section{MATERIALS AND METHODS}

\section{Materials}

Dulbecco's-modified Eagle's medium (DMEM), antibiotic-antimycotic solution, fetal bovine serum (FBS), were purchased from Thermo Fisher. Diphenyleneiodonium chloride (DPI), pramipexole dihydrochloride (PPD), manganese (III) tetrakis (1methyl-4-pyridyl) porphyrin (MnTMPyP), and zinc chloride were purchased from Sigma-Aldrich.

\section{Methods}

\section{Primary culture of cortical astrocytes}

Nowadays, astrocytic injury and death after ischemic brain has gained more and more attention. Reports show that astrocytes can dead before neurons (Liu et al. 1999; Petito et al. 1998). Primary astrocytes were collected from the Sprague-Dawley rats' cortices as described previously (Kim et al. 2010). Briefly, the brains of postnatal day 1 rats were immediately isolated after decapitation. After removing blood vessels and meninges, the forebrains were placed and chopped in DMEM (Thermo Fisher Scientific, USA). After that, the minced tissue was trypsinized in $0.05 \%$ trypsin (Thermo Fisher Scientific, USA) at $37^{\circ} \mathrm{C}$ for $30 \mathrm{~min}$, which was stopped by adding $10 \%(v / v)$ FBS (Thermo Fisher Scientific, USA) in DMEM. Astrocytes were isolated by passing the dissociated tissue through a $40 \mu \mathrm{mol} / \mathrm{L}$ strainer. At last, astrocytes were seeded at a density of $1.5 \times 10^{5}$ cells $/ \mathrm{cm}^{2}$ in growth medium (90\% Dulbecco's Modified Eagle medium containing $10 \% \mathrm{FBS}$, and $4.5 \mathrm{~g} / \mathrm{L}$ glucose). The astrocytes were cultured in incubator at $37{ }^{\circ} \mathrm{C}$ with $95 \%$ air $/ 5 \% \mathrm{CO}_{2}$. Somewhere in this manuscript, you need to justify why you used astrocytes, not neurons, to conduct this study.

\section{Coculture of primary astrocytes and neurons}

Primary astrocytes were cultured on cell culture inserts (BD Biosciences. San Jose, CA) with $0.4 \mu \mathrm{m}$ pores. Primary neurons were isolated from the cortices of E18 Sprague-Dawley rats as previously described (Xu et al. 2012) and cultured on 12 well culture plates. Culture medium of neurons was neurobasal with B27 supplement and $0.5 \mathrm{mmol} / \mathrm{L}$ glutamine solution. The insert with astrocytes was placed into the wells after the neurons had adhered to the wells. Cells were cultured at $37{ }^{\circ} \mathrm{C}$ with $95 \%$ air $/ 5 \% \mathrm{CO}_{2}$. 


\section{Hypoxic cellular model}

Serum-free DMEM has been bubbled with nitrogen to remove oxygen in the medium for $15 \mathrm{~min}$. Then, the oxygen-free medium was used to replace the cell culture medium in a polymer hypoxic glove chamber (Coy Laboratory Products Inc. Grass Lake, MI). The cells, in the oxygen-free medium, were incubated in the hypoxic glove chamber with $1 \% \mathrm{O}_{2} / 99 \%$ gas mixture $\left(5 \% \mathrm{CO}_{2} /\right.$ $95 \% \mathrm{~N}_{2}$ ) at $37{ }^{\circ} \mathrm{C}$. After 3 h hypoxic incubation, the cells were removed from the hypoxic chamber and FBS (final concentration $10 \%$ ) was added to the cells for reoxygenation. Then the cells were placed in cell culture incubator for another $12 \mathrm{~h}\left(5 \% \mathrm{CO}_{2} / 95 \%\right.$ air, $\left.37^{\circ} \mathrm{C}\right)$.

\section{Co-staining of labile zinc with mitochondria}

Astrocytes were seeded onto glass coverslips before hypoxia/reoxygenation treatment. After the treatment, intracellular-free zinc was stained by FluoZin-3 AM (Thermo Fisher, USA), a cell permeable probe with selective fluorescent to stain the labile zinc in cells. Astrocytes were rinsed with DMEM for three times. Then, the cells were incubated with $2.5 \mu \mathrm{mol} / \mathrm{L}$ FluoZin3 in DMEM at room temperature. After 45 min incubation, astrocytes were thoroughly washed with DMEM. Then DMEM containing $100 \mathrm{nmol} / \mathrm{L}$ MitoTracker Red (Thermo Fisher, USA) was used to stain the mitochondria. Following a 30-min incubation, the staining solution was replaced with fresh DMEM. The coverslips were then mounted on a glass slide. Images were acquired using an inverted microscope (Olympus 0X71, Japan) with GFP or TRICT dichroic mirror to visualize FluoZin-3 AM or MitoTracker fluorescence.

\section{Immuno-spin trapping of ROS measurement}

For measurement of ROS in astrocytes, 5,5-Dimethyl-1pyrroline N-oxide (DMPO, Sigma-Aldrich, USA) was used to trap the protein-bound radicals. Astrocytes were plated onto glass coverslips. $100 \mathrm{mmol} / \mathrm{L}$ DMPO was added into culture media before exposing the astrocytes to hypoxia. After $3 \mathrm{~h}$, astrocytes were fixed by a 15-min incubation with 4\% paraformaldehyde (PFA). Following the wash with PBS, cells were immersed in $0.2 \%$ Triton $\mathrm{X}-100$ for $5 \mathrm{~min}$ for permeabilization. Then, 10\% normal goat serum (Thermo Fisher, USA) was used to block the sample. After $1 \mathrm{~h}$ blocking, the sample was incubated with anti-DMPO antibody (Enzo Life Sciences, USA) $\left(10 \mu \mathrm{g} / \mathrm{mL}\right.$, overnight at $\left.4{ }^{\circ} \mathrm{C}\right)$. After three washes with PBS, the coverslips were incubated with Alexa Fluor 488 goat anti-mouse antibody $(2 \mu \mathrm{g} / \mathrm{mL}$, room temperature, ThermoFisher, USA) for $2 \mathrm{~h}$. Then, the fluorescence signal of DMPO-protein was caught by Olympus IX71 fluorescence microscopy using a GFP dichroic mirror.

\section{Cytotoxicity assay}

Astrocytic death was assessed by quantifying the cellular lactate dehydrogenase (LDH) release, using the Cytotox 96 non-radioactive cytotoxicity assay kit (Promega, USA). Astrocytes were sowed in 96-well plates at the density of $5 \times 10^{3}$ cells/well. After the treatments, $50 \mu \mathrm{L}$ reconstituted substrate mixture was added to each well, incubating for $30 \mathrm{~min}$. After the reaction was stop by adding $50 \mu \mathrm{L}$ stop solution to each well, the absorbance at $490 \mathrm{~nm}$ was measured by a microplate reader (Bio-Rad 3350, USA). Triton-X 100 treated cells were used as $100 \%$ cell death control because it releases all intracellular LDH by disrupting their membranes. The cell death rate was calculated by using the formula: Cell death rate $(\%)=($ Experimental absorbance value - Culture medium absorbance value)/ (Triton-X 100 treated absorbance value - Culture medium absorbance value) $\times 100$.

\section{Mitochondria isolation from astrocytes}

$2 \times 10^{7}$ astrocytes were cultured in dishes. After $3 \mathrm{~h}$ of hypoxia exposure, astrocytes were harvested. Mitochondria were isolated using Mitochondria Isolation Kit for Cultured Cells (ThermoFisher, USA) following the user manual.

\section{JC-1 staining for mitochondrial membrane potential of primary astrocytes}

JC-1 was used to estimate the change of mitochondrial membrane potential (Liu et al. 2017). Astrocytes were plated onto glass coverslips. After $3 \mathrm{~h}$ of hypoxia exposure, astrocytes were incubated with $2 \mathrm{mg} / \mathrm{mL} \mathrm{JC}-1$ (Thermo Fisher, USA) at $37{ }^{\circ} \mathrm{C}$ for $20 \mathrm{~min}$. JC-1 green and red were captured by Olympus IX71 fluorescence microscopy using a GFP and TRICT dichroic mirrors.

Acknowledgements This work was supported by National Institutes of Health Grant P30GM103400.

\section{Compliance with Ethical Standards}

Conflict of interest Rong Pan, Ke Jian Liu and Zhifeng Qi declare that they have no conflict of interest.

Human and animal rights and informed consent All institutional and national guidelines for the care and use of laboratory animals were followed. 
Open Access This article is distributed under the terms of the Creative Commons Attribution 4.0 International License (http:// creativecommons.org/licenses/by/4.0/), which permits unrestricted use, distribution, and reproduction in any medium, provided you give appropriate credit to the original author(s) and the source, provide a link to the Creative Commons license, and indicate if changes were made.

\section{References}

Abramov AY, Scorziello A, Duchen MR (2007) Three distinct mechanisms generate oxygen free radicals in neurons and contribute to cell death during anoxia and reoxygenation. J Neurosci 27(5):1129-1138

Adibhatla RM, Hatcher JF (2010) Lipid oxidation and peroxidation in CNS health and disease: from molecular mechanisms to therapeutic opportunities. Antioxid Redox Signal 12(1):125-169

Berry EA, Zhang Z, Bellamy HD, Huang L (2000) Crystallographic location of two $\mathrm{Zn}(2+)$-binding sites in the avian cytochrome bc(1) complex. Biochim Biophys Acta 1459(2-3):440-448

Bitanihirwe BK, Cunningham MG (2009) Zinc: the brain's dark horse. Synapse 63(11):1029-1049

Dineley KE, Votyakova TV, Reynolds IJ (2003) Zinc inhibition of cellular energy production: implications for mitochondria and neurodegeneration. J Neurochem 85(3):563-570

Dineley KE, Richards LL, Votyakova TV, Reynolds IJ (2005) Zinc causes loss of membrane potential and elevates reactive oxygen species in rat brain mitochondria. Mitochondrion 5(1):55-65

Federico A, Cardaioli E, Pozzo PD, Formichi P, Gallus GN, Radi E (2012) Mitochondria, oxidative stress and neurodegeneration. J Neurol Sci 322:254-262

Filipiak M, Bilska E, Tylko G, Pyza E (2010) Effects of zinc on programmed cell death of Musca domestica and Drosophila melanogaster blood cells. J Insect Physiol 56(4):383-390

Galasso SL, Dyck RH (2007) The role of zinc in cerebral ischemia. Mol Med 13(7-8):380-387

Hamatake M, Iguchi K, Hirano K, Ishida R (2000) Zinc induces mixed types of cell death, necrosis, and apoptosis, in molt-4 cells. J Biochem 128(6):933-939

Kim EJ, Raval AP, Hirsch N, Perez-Pinzon MA (2010) Ischemic preconditioning mediates cyclooxygenase-2 expression via nuclear factor-kappa B activation in mixed cortical neuronal cultures. Transl Stroke Res 1(1):40-47

Liu D, Smith CL, Barone FC, Ellison JA, Lysko PG, Li K, Simpson IA (1999) Astrocytic demise precedes delayed neuronal death in focal ischemic rat brain. Brain Res Mol Brain Res 68(1-2):29-41

Liu S, Shi H, Liu W, Furuichi T, Timmins GS, Liu KJ (2004) Interstitial p02 in ischemic penumbra and core are differentially affected following transient focal cerebral ischemia in rats. J Cereb Blood Flow Metab 24(3):343-349

Liu X, Yang L, Long Q Weaver D, Hajnoczky G (2017) Choosing proper fluorescent dyes, proteins, and imaging techniques to study mitochondrial dynamics in mammalian cells. Biophys Rep 3(4):64-72

Martinez-Reyes I, Diebold LP, Kong H, Schieber M, Huang H, Hensley CT, Mehta MM, Wang T, Santos JH, Woychik R, Dufour E, Spelbrink JN, Weinberg SE, Zhao Y, DeBerardinis RJ, Chandel NS (2016) TCA cycle and mitochondrial membrane potential are necessary for diverse biological functions. Mol Cell 61(2):199-209
Nicholls DG, Budd SL (2000) Mitochondria and neuronal survival. Physiol Rev 80(1):315-360

Olmez I, Ozyurt H (2012) Reactive oxygen species and ischemic cerebrovascular disease. Neurochem Int 60(2):208-212

Ouyang YB, Voloboueva LA, Xu LJ, Giffard RG (2007) Selective dysfunction of hippocampal CA1 astrocytes contributes to delayed neuronal damage after transient forebrain ischemia. J Neurosci 27(16):4253-4260

Pan R, Liu KJ (2016) ZNT-1 Expression reduction enhances free zinc accumulation in astrocytes after ischemic stroke. Acta Neurochir Suppl 121:257-261

Pan R, Chen C, Liu WL, Liu KJ (2013) Zinc promotes the death of hypoxic astrocytes by upregulating hypoxia-induced hypoxiainducible factor-1alpha expression via poly(ADP-ribose) polymerase-1. CNS Neurosci Ther 19(7):511-520

Pan R, Timmins GS, Liu W, Liu KJ (2015) Autophagy mediates astrocyte death during zinc-potentiated ischemia-reperfusion injury. Biol Trace Elem Res 166(1):89-95

Petito CK, Olarte JP, Roberts B, Nowak TS Jr, Pulsinelli WA (1998) Selective glial vulnerability following transient global ischemia in rat brain. J Neuropathol Exp Neurol 57(3):231-238

Piantadosi CA, Zhang J (1996) Mitochondrial generation of reactive oxygen species after brain ischemia in the rat. Stroke 27(2):327-331 discussion 332

Poli G, Leonarduzzi G, Biasi F, Chiarpotto E (2004) Oxidative stress and cell signalling. Curr Med Chem 11(9):1163-1182

Ramirez DC, Mason RP (2005) Immuno-spin trapping: detection of protein-centered radicals. Curr Protoc Toxicol. https://doi. org/10.1002/0471140856.tx1707s24

Semenza GL, Agani F, Feldser D, Iyer N, Kotch L, Laughner E, Yu A (2000) Hypoxia, HIF-1, and the pathophysiology of common human diseases. Adv Exp Med Biol 475:123-130

Suh SW, Gum ET, Hamby AM, Chan PH, Swanson RA (2007) Hypoglycemic neuronal death is triggered by glucose reperfusion and activation of neuronal NADPH oxidase. J Clin Investig 117(4):910-918

Towner RA, Smith N, Saunders D, Henderson M, Downum K, Lupu F, Silasi-Mansat R, Ramirez DC, Gomez-Mejiba SE, Bonini MG, Ehrenshaft M, Mason RP (2012) In vivo imaging of immunospin trapped radicals with molecular magnetic resonance imaging in a diabetic mouse model. Diabetes 61(10):2405-2413

Weiss JH, Sensi SL, Koh JY (2000) Zn(2+): a novel ionic mediator of neural injury in brain disease. Trends Pharmacol Sci 21(10):395-401

Xu SY, Wu YM, Ji Z, Gao XY, Pan SY (2012) A modified technique for culturing primary fetal rat cortical neurons. J Biomed Biotechnol 2012:803930

Yang X, Wu J, Jing S, Forster MJ, Yan LJ (2018) Mitochondrial protein sulfenation during aging in the rat brain. Biophys Rep 4(2):104-113

Zamzami N, Kroemer G (2001) The mitochondrion in apoptosis: how Pandora's box opens. Nat Rev Mol Cell Biol 2(1):67-71

Zhao Y, Pan R, Li S, Luo Y, Yan F, Yin J, Qi Z, Yan Y, Ji X, Liu KJ (2014) Chelating intracellularly accumulated zinc decreased ischemic brain injury through reducing neuronal apoptotic death. Stroke 45(4):1139-1147

Zhao Y, Huang Y, Fang Y, Zhao H, Shi W, Li J, Duan Y, Sun Y, Gao L, Luo Y (2018a) Chrysophanol attenuates nitrosative/oxidative stress injury in a mouse model of focal cerebral ischemia/ reperfusion. J Pharmacol Sci 138(1):16-22

Zhao Y, Yan F, Yin J, Pan R, Shi W, Qi Z, Fang Y, Huang Y, Li S, Luo Y, Ji X, Liu KJ (2018b) Synergistic interaction between zinc and reactive oxygen species amplifies ischemic brain injury in rats. Stroke 49(9):2200-2210 